\title{
Limited resources and evolutionary learning may help to understand the mistimed reproduction in birds caused by climate change
}

\author{
Daniel Campos ${ }^{\mathrm{a}, *}$, Josep E. Llebot ${ }^{\mathrm{b}}$ and Vicenç Méndez ${ }^{\mathrm{b}}$

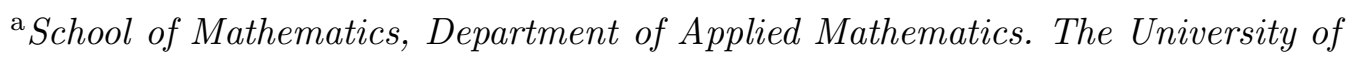 \\ Manchester, Manchester M60 1QD, UK. \\ ${ }^{\mathrm{b}}$ Grup de Física Estadística. Departament de Física. Universitat Autònoma de \\ Barcelona, 08193 Bellaterra (Barcelona) Spain
}




\begin{abstract}
We present an agent-based model inspired by the Evolutionary Minority Game (EMG), albeit strongly adapted to the case of competition for limited resources in ecology. The agents in this game become able, after some time, to predict the a priori best option as a result of an evolution-driven learning process. We show that a self-segregated social structure can emerge from this process, i.e., extreme learning strategies are always favoured while intermediate learning strategies tend to die out. This result may contribute to understanding some levels of organization and cooperative behaviour in ecological and social systems. We use the ideas and results reported here to discuss an issue of current interest in ecology: the mistimings in egg laying observed for some species of bird as a consequence of their slower rate of adaptation to climate change in comparison with that shown by their prey. Our model supports the hypothesis that habitat-specific constraints could explain why different populations are adapting differently to this situation, in agreement with recent experiments.
\end{abstract}

Key words: Evolutionary Learning, Climate Change, Limited Resources, Predator-Prey

* Corresponding author.

Email address: Daniel. Campos@uab.es (Daniel Campos). 


\section{Introduction}

Minority games (Challet and Zhang, 1998), and more recently Evolutionary Minority Games (EMG) (Johnson et. al., 1999a; Johnson et. al., 2000; de Cara et. al., 2000; Johnson et. al., 2003; Hod and Nakar, 2002; Hod, 2003; Sysi-Aho et. al., 2003; Johnson et. al., 1999b; Lo et. al., 2000), have received widespread attention in recent years as a useful model to describe competition for highly limited resources in complex systems, especially in economics. These games are essentially based on a minority rule (Challet and Zhang, 1998) according to which $N$ agents compete repeatedly for some resources by choosing between two options A or B. Each agent makes its choice, and those agents belonging to the less (most) frequently chosen option are considered the winners (losers), so they are rewarded (fined). So, the idea behind this game is that the agents must always try to be in the minority: few individuals choosing the same option as yourself means less competitors, and so it should be easier to obtain the resource. The decisions taken by the agents are chosen according to a pool of strategies available, and these strategies are based on the $m$ previous outcomes in the game, as that information is assumed to be accessible to all of the agents. To give a simple example, a specific strategy in a minority game with $m=2$ has the form

$$
S=\{(A, A) \rightarrow A,(A, B) \rightarrow B,(B, A) \rightarrow A,(B, B) \rightarrow A\}
$$

This means that if the two previous winning options in the game were $(\mathrm{A}, \mathrm{A})$, an agent following strategy $S$ will choose option A the next time; if the last winning options were $(\mathrm{A}, \mathrm{B})$, that agent will choose $\mathrm{B}$, and so on. At the beginning of the game several strategies are assigned to each agent, and the agent tends to choose from among them the strategy that gave better results 
in the past; however, many different versions of the minority game exist, where the rules that determine the strategies chosen by the agents are different. Here, we will skip the minor details on the mechanisms of the minority game, since that is outside the scope of the current work; an exhaustive compilation of works on minority games can be found in http://www.unifr.ch/econophysics.

In the evolutionary version (EMG) of the game (Johnson et. al., 1999a), all the agents are assigned the same strategies but they can $i$ ) follow that given strategy with probability $p_{k}$ or ii) do exactly the opposite with probability $1-p_{k}$, where $p_{k}$ is different for each agent (the subindex $k$ denotes the $k$ th agent). Those agents performing the worst (losing many times) are forced to change their value of $p_{k}$; so, in the EMG there is an implicit learning process based on trial and error. As a consequence, the system tends towards an optimal distribution of $p_{k}$ values for which the number of winners is as close to $N / 2$ as possible (note that, by definition, in a minority game the number of winners cannot be higher than $N / 2$ ). As reported in (Johnson et. al., 1999a), the most striking result arising from the EMG is the natural emergence of segregated behaviour: those agents that behave in an extreme way $\left(p_{k} \rightarrow 0\right.$ and $p_{k} \rightarrow 1$ ) perform better than those with intermediate behaviour, so that the individuals tend to segregate into two groups: those who always follow the given strategy and those who never follow the strategy. From the point of view of complex systems, it has been claimed that this result may help to understand some levels of organization such as crowding (Johnson et. al., 2000; Cont and Bouchaud, 2000) and cooperation (de Cara, 2000), which are common in many social and biological systems. Specifically, within the context of the EMG some authors have coined the term unintentional or indirect cooperation to illustrate the behaviour observed (Quan et. al., 2003; 
Hod and Nakar, 2004). This concept refers to the fact that in the EMG many agents tend to behave similarly (either $p_{k} \rightarrow 0$ or $p_{k} \rightarrow 1$ ), but not consciously, but rather because the global winning probability is higher that way. This is different from other games (for example, the well-known Prisoner's Dilemma) where cooperation is a conscious option given to the agents (Nowak et. al., 2004; Nowak 2006).

\section{Minority Games in ecology}

In general, minority games are helpful to describe multi-agent systems where each agent (individual) is able to analyze the history of the system (i.e., the success of the different strategies used before) in order to make its next decision. For this reason they have been especially designed and used to explain the complex dynamics of some financial markets (Johnson et. al., 2003), albeit some authors have stated that similar ideas could also hold within an ecological context; probably the best example being foraging behaviour (Hod and Nakar, 2002; Hod, 2003). However, as far as we know very few real efforts have been made to extend minority games to ecological situations. In (Tella et. al., 2001) the authors presented a model, inspired by the rules of the minority game, to explore the colonial versus solitary behaviour in birds as a function of predation pressure, and some discussions on the connection between minority games and ecological evolution were provided in (de Cara et. al., 2000; Aho et. al., 2003).

The apparent lack of interest by ecologists in these games is probably due to the fact that the most interesting and dramatic situations concerning decisionmaking in animals are not well described by such concepts as trial and error 
and pool of strategies involved in minority games. Instead, in ecology most of the interest lies in understanding those situations where individuals perform just one or a few critical decisions throughout their whole life (concerning, for example, timing in reproduction or choice of habitat); these decisions have been called 'fitness-critical actions' in a very recent work by Heesch and Little (2006). Intuitively, decision-making in these 'fitness-critical actions' follows quite simple mechanisms (compared to the complex rules of minority games): the individuals need to use their skills or their experience to predict the $a$ priori best option. By a priori best option we mean that option which would be the winning one in the case where half of the agents choose $\mathrm{A}$ and the other half choose B. In the basic minority game described above we have considered that the agents choose between two identical options $\mathrm{A}$ or $\mathrm{B}$, so there is no a priori best option. However, it is easy (and more realistic) to consider a game where A and B are intrinsically different. For example, in the case of habitat selection, individuals usually need to choose between different options with different habitat qualities. Some individuals may be able, from past experience, to know in advance which the best choice is e.g. that where the availability of food is higher. But if all the individuals are able to do this, then all of them will choose the same option and the availability of food will decrease there; in that case the a priori best option is not necessarily the winning option. Those individuals that are not able to determine what the $a$ priori best option is will probably behave randomly or persistently (always choosing the same option). The role of evolution and natural selection is thus expected to be crucial in these processes, as stated in (Heesch and Little, 2006).

We note that these decision-making mechanisms are also common in human 
behaviour. For instance, drivers who have to choose between two alternative routes in order to avoid traffic jams do not analyze every past experience and make a decision according to a pool of strategies (contrary to what is suggested by some authors (Hod, 2003)), but mainly use simpler strategies like persistent behaviour (they always choose the same route because they do not like to take risks) or they may simply listen to the traffic news to find out what the a priori best route is.

According to these arguments, some essential elements which are absent in the EMG must be considered in order to get a realistic implementation of minority games in ecology. So, the aim of this work is to present a new game where competition for resources is also introduced by means of a minority rule, but the dynamics and strategies followed by the agents aim to capture the dynamics of some ecological systems. In what follows, we will refer to this new model as the Evolutionary Learning Game (ELG).

\section{Mistiming in predator-prey systems caused by climate change}

We now introduce a specific problem that has attracted the interest of ecologists in recent years (van Noordwijk et. al., 1995; Visser et. al., 1998; Grieco et. al., 2002; Visser et. al., 2004; Gienapp and Visser, 2006) and has strongly motivated our approach. In many species of bird, individuals must face the problem of choosing the correct time for egg laying. This choice becomes dramatic if the availability of food is restricted to a very short period of time. So, for survival in breeding, the correct timing of egg laying is necessary, so that the feeding period matches the food peak. This process has been studied in recent decades for some species, such as great tits (Parus major) and 
blue tits (Parus caeruleus), whose main prey (caterpillar) is only available for two or three weeks in the late spring (Visser et. al., 2004). At the moment of egg laying (approximately one month before), the birds do not know when the food peak will happen. The problem is partially overcome by the way many of these birds develop with age the ability to follow some cues (based on climate and other environmental parameters) to predict the right time for laying (van Noordwijk et. al., 1995; Grieco et. al., 2002; Gienapp and Visser, 2006). In general, this capacity of an individual to adapt its behaviour to the environmental conditions is known as phenotypic plasticity, and is usually a heritable trait. Specifically, it has been demonstrated (Nussey et. al., 2005) that plasticity in egg laying for birds is heritable.

The effects of global climate change, however, have put many biological species to the test (Parmesan, 2006). As a consequence of warmer springs, caterpillars have advanced their hatching date in many habitats (Visser et. al., 1998; Visser et. al., 2004), so those birds with a higher plasticity in laying are expected to adapt better to the new situation. According to the observational data, some bird populations have become adapted, but in some other cases a very weak response to the new situation has been observed (Visser et. al., 2004; Gienapp and Visser, 2006). In the latter case, the mismatching between the feeding period and the food peak will probably lead to a decline in the number of individuals (Both et. al., 2006) or the habitat fitness (Visser, 2007). Although different explanations have been provided, there is no clear understanding of why different populations show different responses to the changing conditions (Gienapp and Visser, 2006). As we discuss below, our model provides some arguments that support the idea that resource constraints from each specific habitat may be responsible for these differences. 


\section{Rules of the Evolutionary Learning Game}

We need to introduce two basic ideas that are missing from the original formulation of the EMG, in order to reach a more realistic description of ecological systems:

i) First, reproduction and death processes must play a fundamental role in the dynamics of the system. In the EMG the agents continue to play indefinitely, but in ecology the consequences of choosing a wrong option can obviously be dramatic. If we want to explore the dynamics of systems over representative time scales, it is necessary to assume that the individuals may disappear (die) and/or be replaced by new individuals (newborns) with some probability. Moreover, the outcome obtained from any decision taken by the agents must affect in some way their reproduction/survival probabilities.

ii) Secondly, in the EMG all of the agents have access to the same information, and so all of them may use the same strategy. In ecology, however, as long as an individual grows up it gains experience and, in consequence, it is expected to choose better options. So, an individual learning process must be considered somehow. In fact, the situation where the strategies chosen by the agents are based on their individual histories has already been studied for the EMG (see (de Cara, 2000) and the references therein), but here we will explore the concept of learning from a different perspective.

In our model, each of the $N$ agents competing in the game must repeatedly choose between options A or B. Whether or not the decision taken by the agent is the good one will be determined by a minority rule with an arbitrary cutoff, as defined in (Johnson et. al., 1999b). This means that we assign a resource 
capacity $L$ to one of the two options (we consider $0<L<N / 2$ without loss of generality) and a resource capacity $N-L$ to the other option. If the real number of agents choosing the first option is below $L$, then the resources per capita in that option are higher than in the other one, so those agents are the winners and the agents choosing the other option are the losers. If the number of agents choosing the first option is above $L$, then the contrary arguments hold.

We will consider that the option with capacity $L$ is not always the same, but is chosen randomly every time step in order to incorporate the effects of a fluctuating environment, so sometimes option A will be the a priori best option (that with a higher capacity) and sometimes not.

At each time step, the winners are rewarded with the possibility of reproductive success. Every winner is given the possibility of producing a newborn agent with a probability $r$. The newborn will replace one of the agents in the game (to keep $N$ constant) chosen randomly, so we assume that all of the agents are equally likely to die.

The agents choose option A or B according to the following rules. Younger agents act persistently: they make their first choice randomly and, after that, they continue to choose the same option. However, after each time step all the persistent agents are given the possibility of learning with probability $p p_{k}$ (here $p p$ stands for phenotypic plasticity and the subindex $k$ denotes the $k$ th agent). If they learn, it means that they give up persistent behaviour; from then on, they always choose the option with a higher capacity, so we will say that they become wise agents.

Phenotypic plasticity in our model is thus considered equivalent to a learning 
capacity. This capacity can be inherited as follows: when a newborn appears, its characteristic probability $p p_{k}$ is chosen randomly from an interval of width $w$ centred on the value of $p p_{k^{\prime}}$ from its father, with reflecting boundary conditions at $p p_{k}=0$ and $p p_{k}=1$. So, we introduce an evolutionary dynamics for the probabilities $p p_{k}$ into the model in a similar way as in the original formulation of the EMG (Johnson et. al., 1999a). But note that in this case the meaning of the width $w$ is extremely important for the dynamics of the system, as it measures the heritability of $p p_{k}$, so the best strategies $p p_{k}$ will be transmitted to the newborns only if $w$ is not too high.

These are all the rules for our ELG. All of the agents will become wise sooner or later unless they die first, but if there are too many wise agents then the a priori best option will be crowded and will probably be the wrong one. A complex dynamic thus emerges where learning as fast as possible is not necessarily the best strategy, which may seem counterintuitive at first.

As some of the rules presented could be considered too simple or unrealistic from a biological point of view, we tried to implement many different models with increasingly complex rules in order to compare their performances:

i) We tried to introduce explicit reproduction and death algorithms in many different ways (for example, by using exponential or logistic growth), so that the number of agents $N$ was allowed to change with time.

ii) We tried to replace the minority rule with some other competition rules, even rules that allowed all the agents to be winners (or losers) at the same time. For instance, we considered two independent capacities $L_{A}$ and $L_{B}$ for the two possible options, so if the number of individuals choosing option $\mathrm{A}$ is above (below) $L_{A}$ those agents are considered losers (winners). 
iii) We tried to reward and/or fine agents on their reproductive success and/or their probability of survival. Of course, it is not necessary for the reproductive success to be completely suppressed for the losers as in the simplified version we have described; we could consider two reproductive rates $r_{w}$ and $r_{l}$ for winners and losers respectively, with $r_{w}>r_{l}$.

iv) We tried to consider that the switching from persistent to wise behaviour is not so radical, but the agents learn progressively according to a rate given by $p p_{k}$.

After these and many other trials, we have found that the qualitative behaviour exhibited by the ELG (which is shown in the following Section) is highly robust. According to our results, it seems that there are only two elements which are strictly necessary in order to obtain that behaviour: i) a learning process regulated by the probabilities $p p_{k}$ and ii) that the number of agents rewarded (fined) is proportional to the number of winners (losers). The version of the ELG we have presented here is one of the simplest possible, and so it offers the advantage that some analytical treatment is possible, as we will show below.

\section{$5 \quad$ Results}

The greatest interest of our model lies in the form of the distribution of phenotypic plasticities $P\left(p p_{k}\right)$ that is reached in the steady state. In Figure 1 we summarize the behaviour of $P\left(p p_{k}\right)$ as a function of the three parameters of the model: $L, r$ and $w$. All the results shown here were obtained by computing the form of $P\left(p p_{k}\right)$ for $N=2001$ after 10000 time steps (which is far enough to 
reach the steady state), and carried out an average of 25 different realizations. Initially all the agents were considered newborns and the values of $p p_{k}$ were assigned randomly; anyway, we have checked that our results are independent of the initial conditions chosen.

The series of plots from 1.a to 1.d shows how $P\left(p p_{k}\right)$ changes when the value of $L$ is modified. Figures 1.a and 1.d correspond to extreme situations that are clearly predictable. In the first case, when $L \rightarrow N / 2$ both options A and B have similar resource capacities. Therefore, performing as a wise agent does not represent an advantage, because the resource of choice (i.e. the larger one) reaches its smallest possible size leading to overcrowding among wise agents. As a consequence, learning is avoided and there is a tendency $p p_{k} \rightarrow 0$. In the regime $L \rightarrow 0$ one of the two options is much better than the other one. In this situation, performing as a wise agent is a strong advantage, and so the tendency $p p_{k} \rightarrow 1$ should be expected. But, surprisingly, there is a wide range of intermediate values of $L$ where segregated (obviously asymmetric) behaviour is found. This means that in intermediate situations the dynamics of the system tends to favour individuals which either learn as fast as possible or avoid learning as much as possible.

Although segregated behaviour was also found for the EMG, the situation reported here is clearly different. In the case of the EMG (Johnson et. al., 1999a) the segregated behaviour in the steady state was independent of the initial conditions and the values of the parameters introduced. However, Hod and Nakar (2002) proved later that the model is extremely sensitive to the prize-to-fine ratio, so for some parameters one observes a sharp transition where self-segregation is destroyed. On the contrary, we have not noticed such effects in our model, but the form of $P(p p k)$ always changes smoothly for 
any region of parameters considered. The other main difference between the EMG and the ELG is that the results obtained here show an asymmetric distribution of $P\left(p p_{k}\right)$. The reason for this is that in the ELG the persistent and wise agents do not necessarily choose different options (while in the EMG $p_{k} \rightarrow 0$ and $p_{k} \rightarrow 1$ represent opposite behaviours). This, together with the different backgrounds considered and some ideas discussed below, shows that the general dynamics of the EMG and the ELG are different, although there are some major similarities between both.

One can observe in the series 1.e to $1 . h$ the role of the reproduction probability $r$ on $P\left(p p_{k}\right)$ while keeping the other parameters constant. A high value of $r$ involves the appearance of many newborns and, according to the discussion above, a wise strategy will then perform better that in a situation with few newborns. For this reason, the model shows a tendency $p p_{k} \rightarrow 0$ for low $r$ and a tendency $p p_{k} \rightarrow 1$ for high $r$. In intermediate situations, a segregated distribution is found again.

Finally, the role of $w$ is shown in plots from $1 . i$ to 1.l. As discussed above, the value of $w$ determines the heritability of the phenotypic plasticity. Low values of $w$ represent a high level of heritability and so the best strategies persist, while a high value of $w$ means that best strategies are not well transmitted to breeding; so, for the latter $P\left(p p_{k}\right)$ is expected to tend to be uniform. The value of $w$ also indirectly affects the number of winners and losers; if $w$ is too high the best strategies do not persist and then the average number of winners decreases. For this reason it is difficult to predict the exact form of $P\left(p p_{k}\right)$ as a function of $w$. Actually, the specific role of $w$ in the game is fairly complicated, so this point will be addressed in detail in a further study. Note that this is another important difference from the case of the EMG, where the 
final distribution $P\left(p_{k}\right)$ is almost independent of $w$ (Johnson et. al., 1999a).

We can give some analytical support to the results shown in Figure 1 by means of a mean-field-like approach as proposed before for the EMG (Lo et. al., 2000). Here we keep as much as possible to the notation used there in order to facilitate understanding.

First of all, note that we know the option that the wise agents will choose. We also know that every persistent agent made its first choice randomly, so we should expect on average for half of them to choose A and the other half to choose B. Therefore, the whole problem is reduced to finding out how many persistents are in the game. We denote $F_{N}(n)$ as the probability of $n$ of the $N$ agents in the game being persistent. Similarly, we define $G_{N-1}^{k}(n)$ as the probability of $n$ of the agents being persistent, given that the $k$ th agent is the only one that has not made its choice yet. Then, the following relation holds:

$$
F_{N}(n)=\Gamma_{p p_{k}} G_{N-1}^{k}(n-1)+\left(1-\Gamma_{p p_{k}}\right) G_{N-1}^{k}(n)
$$

where $\Gamma_{p p_{k}}$ is the probability of the $k$ th agent being persistent, given that its phenotypic plasticity is $p p_{k}$. In the following, we will use the simplified notation $\Gamma \equiv \Gamma_{p p_{k}}$.

On the other hand, the winning probability $\tau_{p p_{k}}$ of an agent that has a plasticity $p p_{k}$ can be written as

$$
\tau_{p p_{k}}=\frac{\Gamma}{2} \sum_{n=0}^{\alpha-1} G_{N-1}^{k}(n)+\left(1-\frac{\Gamma}{2}\right) \sum_{n=\alpha+1}^{N-1} G_{N-1}^{k}(n)
$$

where $\alpha=\operatorname{Int}(2 L)$ denotes the integer part of $2 L$. Following now the same treatment as in (Lo et. al., 2000), we get from (11) and (2) the expression 


$$
\begin{aligned}
\tau_{p p_{k}}= & \frac{\Gamma}{2} \sum_{n=0}^{\alpha} F_{N}(n)+\left(1-\frac{\Gamma}{2}\right) \sum_{n=\alpha+1}^{N-1} F_{N}(n) \\
& +\Gamma(\Gamma-3 / 2) G_{N-1}^{k}(\alpha) .
\end{aligned}
$$

As stated in (Lo et. al., 2000), the two first terms correspond to the winning probability of a $k$ th agent whose action does not modify the result of the game, once the other $N-1$ agents have made their choice. Hence, the third term is the essential one, as it measures the influence of the $k$ th agent on the final result. For example, imagine that after the first $N-1$ agents have made their choice, one half of them decide to follow option A (so one half choose B); then, the last agent?s decision will determine which the winning option is; the influence of this final decision on the probability $\tau_{p p_{k}}$ is what the last term in (3) measures. In order to analyze this term, we need to find the explicit expression of $\Gamma$ as a function of $p p_{k}$. This is easy to do, because after each time step the persistents have a probability $p p_{k}$ of becoming wise agents and an average survival probability $s$ (according to the rules of our ELG, only the range $0.5<s<1$ holds here). Then, the probability of the $k$ th agent being persistent is

$$
\Gamma_{p p_{k}}=\frac{\sum_{i=0}^{\infty}\left[s^{i}\left(1-p p_{k}\right)^{i}\right]}{\sum_{i=0}^{\infty} s^{i}}=\frac{1-s}{1-s\left(1-p p_{k}\right)} .
$$

Now, we can give an explicit expression for the term $\Gamma(\Gamma-3 / 2)$ from Equation (3). It can be seen that $\Gamma(\Gamma-3 / 2)$ has the same appearance throughout the whole range $0<p p_{k}<1$ and for the proper range of survival probabilities $0.5<s<1$. It is always a negative convex function with a relative minimum at $p p_{k}=(1-s) / 3 s$. This means that the third term in (3) always tends to favour extreme values of $p p_{k}$, which facilitates the emergence of segregated behaviour; so, this gives some justification to our numerical results. 
We stress, however, that stationary analytical approaches such as the one used here have some limitations, as the EMG and similar models never reach a true stationary distribution (Hod, 2002). In fact, we have found for the ELG that the number of persistents oscillates periodically over time, in accordance with similar results found for the EMG (Hod, 2002). In that work, it was also argued that when the amplitude of these oscillations increases, we observe in the EMG a transition from self-segregated behaviour to clustering (where clustering is characterized by a single-peaked distribution of $p_{k}$ values around $p_{k}=1 / 2$ ). It is interesting to note that, on the contrary, in the ELG the amplitude of the oscillations increases in the region where self-segregated behaviour is found; so, the oscillations in our model seem to enforce self-segregation rather than destroying it. This situation is shown in Figure 2, where the number of persistents in the steady regime is plotted as a function of time for different situations; dotted, dash-dotted and solid lines correspond to the cases 1.a, 1.c and 1.d reported in Figure 1, respectively. From Figure 2, it is also clear that the mean number of persistents in the game increases as $L$ decreases, in accordance with our discussion above.

\section{Discussion}

We have presented a model that sets the ideas of minority games, which are considerably popular tools for describing competition for resources in economics, into an ecological context. The model presented here shows how social segregation emerges from an evolutionary learning process (determined by the distribution $P\left(p p_{k}\right)$ ) in a group of individuals competing for strongly limited resources. Note that if the learning process were not introduced to the model, 
then the dynamics of the system would be trivial. Neither persistent nor wise behaviour on its own is an efficient strategy; evolutionary learning is the key ingredient here for finding efficient cooperation between both. This idea, together with the robustness shown by our model (many other implementations with more realistic rules led to similar qualitative results) seems to suggest that our model could be of interest for understanding social organization in complex evolutionary systems.

The results obtained here show that in the situation described by our model intermediate learning strategies cannot persist for long times; the tendency is always towards improvement $\left(p p_{k} \rightarrow 1\right)$, suppression $\left(p p_{k} \rightarrow 0\right)$ or the coexistence of both (segregated distribution). It also contradicts the intuitive idea that those individuals with a higher learning capacity must always be favoured by selection. This is a consequence of the strong competition process which is assumed in our ELG and in minority games in general: sometimes the a priori worst option can be the best because many agents tend to choose the a priori best option and then competition for the latter is higher.

Finally, we come back to the problem of egg laying in birds described before, which can now be addressed using the ideas about learning and phenotypic plasticity discussed here. In order to provide an analogy with our model, we could imagine that option A means laying early and option B means laying later. Those individuals with a higher plasticity very quickly become able to follow some environmental cues in order to predict the right option $\mathrm{A}$ or $\mathrm{B}$. However, if the penalty for choosing a wrong option is not high (because there are some other food resources available, or there are some other environmental constraints on laying...) then selection will not favour individuals with higher plasticity. In that case, when the individuals must face sudden environmen- 
tal changes their capacity to respond will be weak. In those habitats where phenotypic plasticity is strongly rewarded (i.e., for $L$ small in our model), individuals are expected to follow efficient learning strategies, so they will be able to respond better to environmental changes.

Therefore, the results of our model provide an evolutionary basis to the idea that environmental constraints from each specific habitat could explain why different bird populations are responding differently to climate-driven changes in the behaviour of their prey, as recent experiments have suggested (Gienapp and Visser, 2006). However, empirical evidence supporting our ideas about learning and phenotypic plasticity is still lacking. We believe that it would be of major interest if experimentalists were to attempt to check the predictions made by our model in real ecological systems.

\section{Acknowledgements}

This research has been partially supported by the Generalitat de Catalunya through grant 2006-BP-A-10060 (DC), by the project CGL 2007-60797 (JELl) and by grants FIS 2006-12296-C02-01, SGR 2005-00087 (VM).

\section{References}

Both, C., Bouwhuis, S., Lessells, C.M., Visser, M.E., 2006. Climate change and population declines in a long-distance migratory bird. Nature 441, 81-83.

Challet, D., Zhang, Y.C., 1998. Emergence of cooperation and organization in an evolutionary game. Physica A 246, 407-418; On the minority game: 
Analytical and numerical studies. Physica A 256, 514-532.

Cont, R., Bouchaud, J.P., 2000. Herd behavior and aggregate fluctuations in finanical markets. Macroeconom. Dyn. 4, 170-196.

de Cara, M.A.R., Pla, O., Guinea, F., 2000. Learning, competition and cooperation in simple games. Eur. Phys. J. B. 13, 413-416.

Gienapp, P., Visser, M.E., 2006. Possible fitness consequences of experimentally advanced laying dates in Great Tits: differences between populations in different habitats. Funct. Ecol. 20, 180-185.

Grieco, F., van Noordwijk, A.J., Visser, M.E., 2002. Evidence for the effect of learning on timing of reproduction in blue tits. Science 296, 136-138.

Heesch, D., Little, M., 2006. Decision-making in variable environments - a case of group selection and inter-generational conflict? Theor. Pop. Biol. 69, $121-128$.

Hod, S., 2003. Time-dependent random walks and the theory of complex adaptive systems. Phys. Rev. Lett. 90, 128701-4.

Hod, S., Nakar, E., 2002. Self-segregation versus clustering in the evolutionary minority game. Phys. Rev. Lett. 88, 238702-4.

Hod, S., Nakar, E., 2004. Evolutionary minority game: The roles of response time and mutation threshold. Phys. Rev. E 69, 066122.

Johnson, N.F., Hui, P.M., Jonson, R., Lo., T.S., 1999. Self-Organized segregation within an evolving population. Phys. Rev. Lett. 82, 3360-3363.

Johnson, N.F., Hui, P.M., Zheng, D., Thai, C.W., 1999. Minority game with 
arbitrary cutoffs. Physica A 269, 493-502.

Johnson, N.F., Leonard, D.J.T., Hui, P.M., Lo., T.S., 2000. Evolutionary freezing in a competitive population. Physica A 283, 568-574.

Johnson, N.F., Jefferies, P., Hui, P.M., 2003. Financial market complexity. Oxford University Press, Oxford.

Lo, T.S., Hui, P.M., Johnson, N.F., 2000. Theory of the evolutionary minority game. Phys. Rev. E 62, 4393-4396.

Nowak, M.A., Sasaki A., Taylor, C., Fudenberg, D., 2004. Emergence of cooperation and evolutionary stability in finite populations. Nature 428, 646-650.

Nowak, M.A., 2006. Five Rules for the Evolution of Cooperation. Science 314, $1560-1563$.

Nussey, D.H., Postma, E., Gienapp, P., Visser, M.E., 2005. Selection on heritable phenotypic plasticity in a wild bird population. Science 310, 304-306.

Parmesan, C., 2006. Ecological and evolutionary responses to recent climate change. Annu. Rev. Ecol. Evol. Syst. 37, 637-669.

Sysi-Aho, M., Chakraborti, A., Kaski, K., 2003. Biology helps you to win a game. Phys. Scr. T106, 32-35.

Tella, J.A., de Cara, M.A.R., Pla, O., Guinea F., 2001. A model for predation pressure in colonial birds. In Sixth Granada Lectures in Computational Systems. Ed. Garrido, P.L., Marro, J. AIP Conf. Proc. 574. AIP, New York.

van Noordwijk, A.J., McCleery, R.,Perrins, C., 1995. Selection for the timing of great tit breeding in relation to caterpillar growth and temperature J. Anim. 
Ecol. 64, 451-458.

Visser, M.E., van Noordwijk, A.J., Tinbergen, J.M., Lessells, C.M., 1998. Warmer springs lead to mistimed reproduction in great tits (Parus major). Proc. Royal Soc. London B 265, 1867-1870.

Visser, M.E., Both, C., Lambrechts, M.M., 2004. Global climate change leads to mistimed avian reproduction. Adv. Ecol. Res. 35, 89-110.

Visser, M.E., 2007. Keeping up with a warming world; assessing the rate of adaptation to climate change. Proc. R. Soc. B. DOI 10.1098/rspb.2007.0997.

Quan, H.-J., Wang, B.-H., Hui, P.M., Luo, X.-S., 2003. Self-segregation and enhanced cooperation in an evolving population through local information transmission. Phys. A 321, 300-308. 


\section{Figure captions}

Figure 1.

Plot of the distribution $P\left(p p_{k}\right)$ in the steady state (after 10000 time steps) for $N=2001$ averaged over 25 realizations. The series $a$ to $d$ shows the effect of varying $L$ while keeping $w=0.1$ and $r=0.4$. The series $e$ to $h$ shows the effect of varying $r$ while keeping $L=0.2 N$ and $w=0.1$. The series $i$ to $l$ shows the effect of varying $w$ while keeping $L=0.2 N$ and $r=0.3$.

Figure 2.

Number of persistents as a function of time once in the steady state (the results plotted correspond to times $500<t<1000$ and $N=2001)$. The dotted line corresponds to the parameter values in Figure 1.a.; the dashed-dotted line corresponds to parameter values in Figure 1.c.; the solid line corresponds to the parameter values in Figure 1.d. 


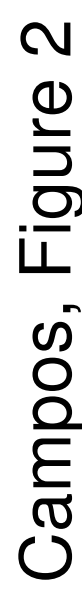

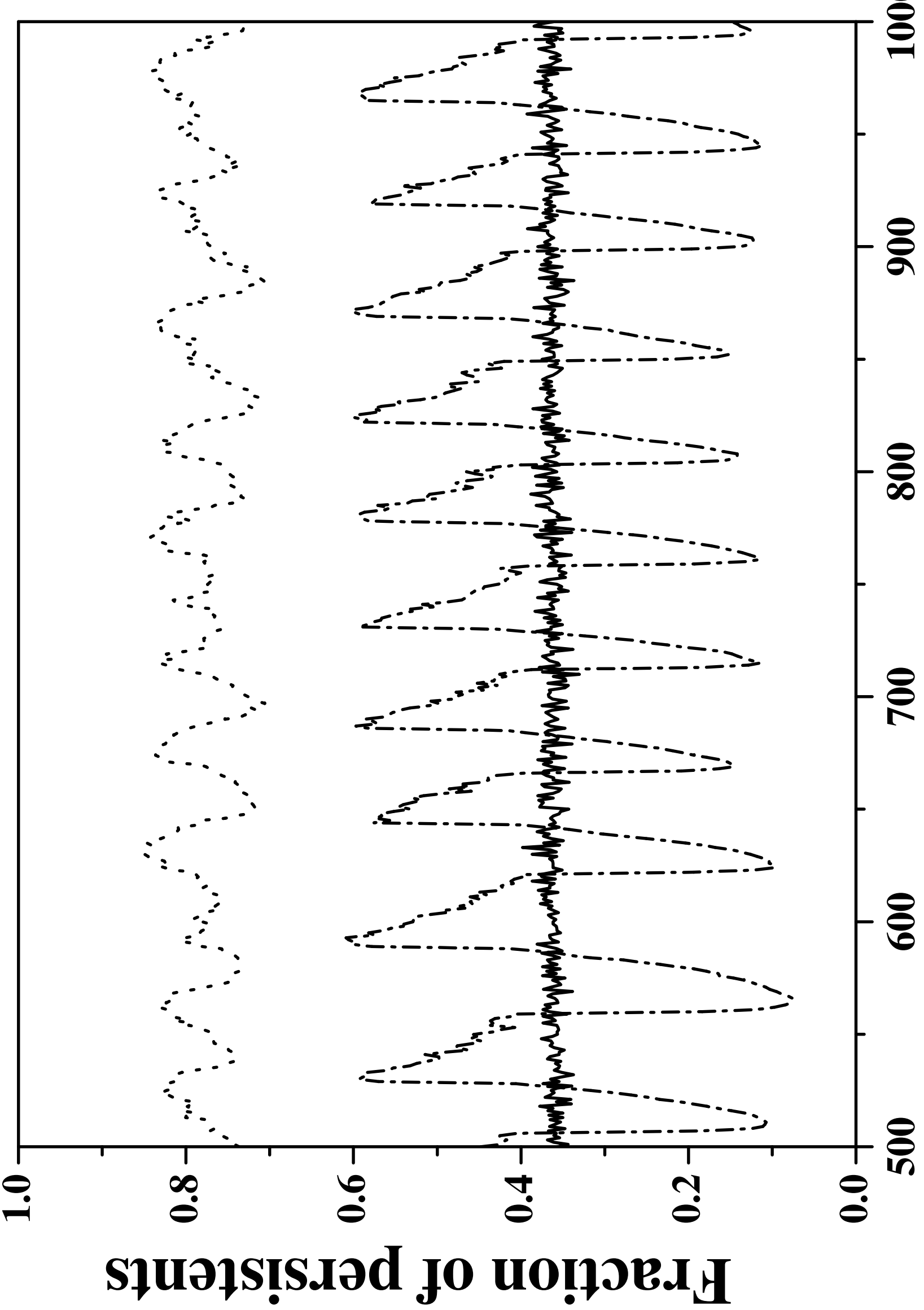



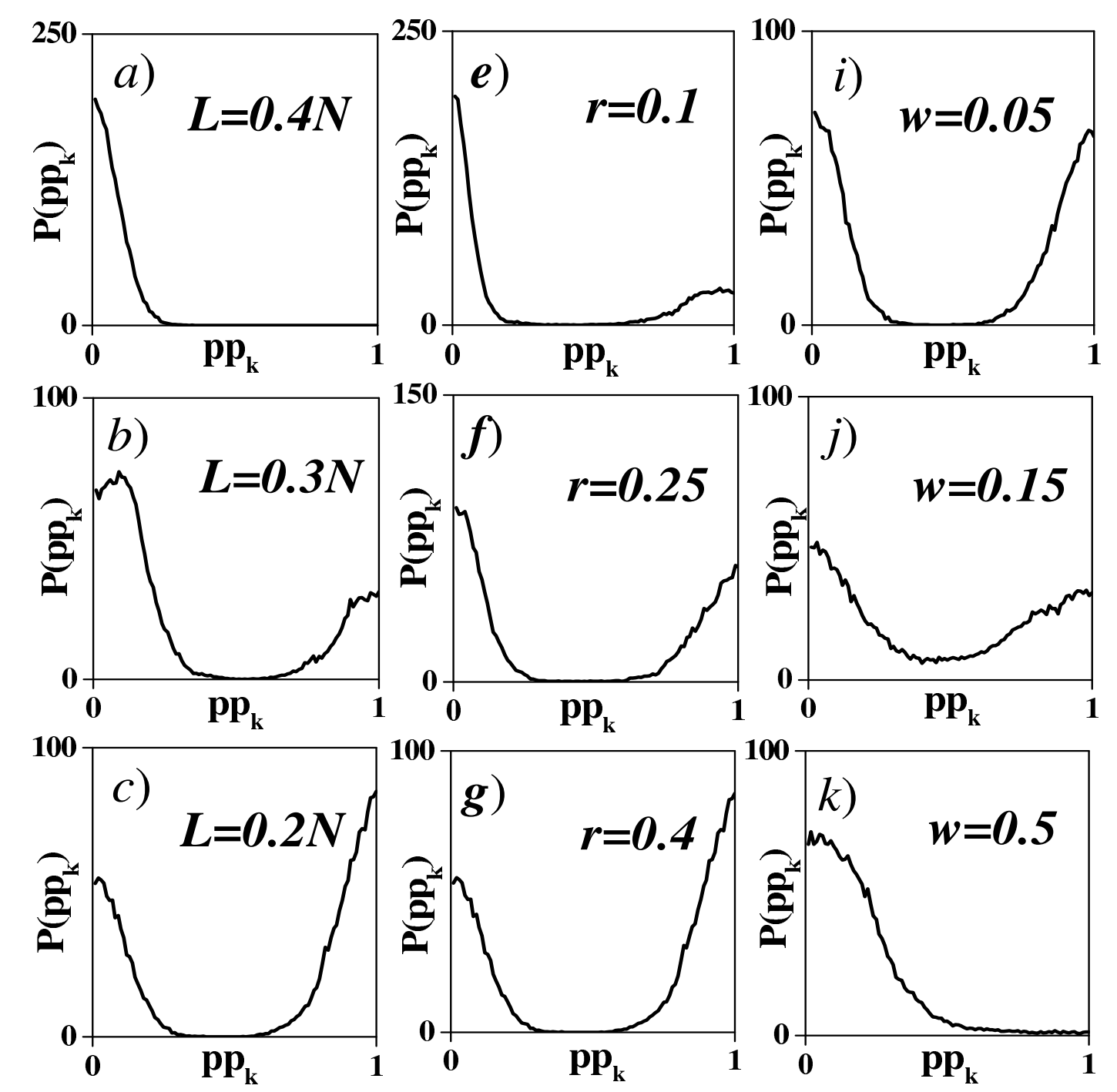

200
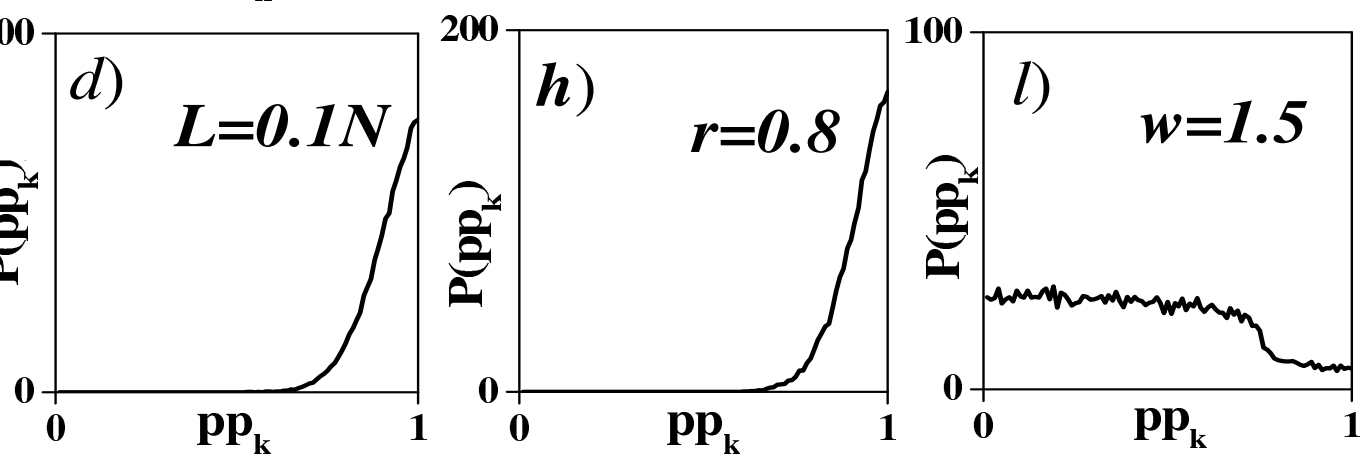

Campos, Figure 1 